\title{
Estado nutricional dos alunos da Rede Nacional de Ensino de Educação Infantil e Fundamental do Serviço Social do Comércio (Sesc), Brasil, 2012
}

\author{
Nutritional status of schoolchildren of the National Child \\ and Youth Education Teaching Network of the Social Service \\ of Commerce (Sesc), Brazil, 2012
}

Luiz Antonio dos Anjos ${ }^{1}$

Willian Dimas Bezerra da Silveira ${ }^{2}$

${ }^{1}$ Laboratório de Avaliação Nutricional e Funcional, Departamento de Nutrição Social, Universidade Federal Fluminense (UFF). R.

Mário Santos Braga 30/415, Centro. 24020-971 Niterói RJ Brasil.lanjos@ig.com.br. ${ }^{2}$ Programa de Pós-

Graduação em Saúde Coletiva, Instituto de Saúde da Comunidade, UFF.

Niterói RJ Brasil.

\begin{abstract}
The objective of this study was to identify and describe the growth and nutritional anthropometric profile of children enrolled in the Sesc National Network of Elementary Education. It is a cross-sectional study conducted among 20,113 students $(9,992$ girls) from 83 schools aged from 3 to 17. Nutritional status was determined using the body mass index for age and stature for age according to the WHO criteria. Stunting was observed in only $1.6 \%$ (girls) and 1.3\% (boys). Overweight + obesity was observed in $29.7 \%$ of the schoolchildren (27.6\% of girls and $32.3 \%$ of boys). In the $<5 y$ schoolchildren, BMI was equal to $12.3 \%$ (10.9\% of girls and $13.2 \%$ of boys). In the 5-10y group, the prevalence was $35.3 \%$ in girls and $40.1 \%$ in boys $(37.8 \%$ in all $)$. In the $10 y+$ group, there were $32.5 \%$ (girls) and $43.0 \%$ (boys), $37.4 \%$ overall. The identified prevalences of BMI in all age groups were high and the problem worsens in older children and in boys. These findings corroborate the reported situation in national and international studies and reinforce the need for monitoring and intervening in the nutritional status of schoolchildren.
\end{abstract}

Key words Obesity, Nutritional status, Preschool, Children, Adolescents
Resumo O objetivo deste estudo foi identificar e descrever o crescimento e o perfil nutricional antropométrico das crianças matriculadas na Rede Nacional de Ensino do Sesc. Trata-se de um estudo transversal realizado em 20.113 escolares (9.992 meninas) em 83 escolas com idades de 3 a 17 anos. $O$ estado nutricional foi determinado pelo indice de massa corporal e estatura para a idade. Os resultados indicaram baixa prevalência de baixa estatura: 1,6\% para meninas e 1,3\% para meninos. No entanto, EMC (excesso de massa corporal) foi encontrado em $29,7 \%$ das crianças, sendo $27,6 \%$ em meninas e $32,3 \%$ em meninos. Nas crianças até 5 anos, EMC foi igual a 12,3\% (10,9\% em meninas e $13,25 \%$ em meninos). $\mathrm{Na}$ faixa etária de 5 a 10 anos, os resultados foram de $35,3 \%$ para as meninas e $40,1 \%$ para os meninos, sendo de $37,8 \%$ em todo o grupo. A prevalência de EMC para crianças acima de 10 anos foi de $37,4 \%$ (32,5\% em meninas e 43,0\% em meninos). As prevalências de EMC encontradas em todas as faixas etárias foram expressivas e o problema se agrava nas crianças maiores e nos meninos. Estes resultados corroboram a situação relatada nos estudos nacionais de alta prevalência de EMC e reforçam a necessidade de acompanhamento do estado nutricional devido aos agravos associados ao EMC infantil e suas consequências na vida adulta. Palavras-chave Obesidade, Estado nutricional, Pré-escolar, Crianças, Adolescentes 


\section{Introdução}

O estado nutricional de crianças e adolescentes representa a condição de vida de uma população e indica sua perspectiva de vida e saúde na vida adulta. Atualmente, percebe-se um antagonismo de tendências na prevalência de desnutrição e obesidade no Brasil, com o declínio da ocorrência da desnutrição em crianças e adultos e aumento do sobrepeso e obesidade em todos os segmentos da população ${ }^{1}$, característica marcante do processo de transição nutricional ${ }^{2}$, fenômeno observado praticamente em todas as populações do mundo ${ }^{3}$.

A Organização Mundial da Saúde (OMS) define obesidade como uma doença caracterizada pelo excesso de gordura corporal que traz prejuízos à saúde ${ }^{4}$, sendo o resultado de um quadro de balanço energético positivo prolongado ${ }^{5}$. Este desequilíbrio decorre da inter-relação entre os fatores genéticos e socioambientais que influenciam diretamente a resposta fisiológica e o comportamento dos indivíduos ${ }^{6}$ e traz consequências sérias à saúde ${ }^{7}$. Considerando-se a complexidade e o elevado custo para o tratamento da obesida$\mathrm{de}^{8}$, sua prevenção constitui-se em ação fundamental no quadro epidemiológico nacional. Por este motivo, a identificação precoce do estado nutricional dos grupos mais vulneráveis permitirá melhor direcionamento das políticas de saúde pública, para minimizar os riscos de doenças crônicas não transmissíveis ligadas à alimentação e ao estilo de vida na vida adulta, ${ }^{9,10}$.

O ambiente escolar tem sido considerado um espaço privilegiado para a realização do levantamento de dados para a avaliação nutricional ${ }^{11}$, bem como para as intervenções necessárias. Considerando sua abrangência e capilaridade, com escolas em 24 estados brasileiros, a Rede Nacional de Ensino de Educação Infantil e Fundamental (RENEIF) do Serviço Social do Comércio (Sesc) contribui de forma marcante para o sistema educacional nacional. Nesta perspectiva, o objetivo do presente estudo é descrever o estado nutricional dos escolares da RENEIF do Sesc segundo sexo, idade e regiões do país. Esta atividade se constitui no primeiro passo para a implantação de um sistema de vigilância nutricional nacional na RENEIF do Sesc.

\section{Materiais e Métodos}

\section{Campo, Delineamento e População do estudo}

O Sesc foi criado em 13 de setembro de 1946, como resultado da ação de empresários e organizações administradas por empresários para atender "às necessidades sociais urgentes"12 dos trabalhadores no comércio, hoje também, de bens e serviços, como instrumento de transformação e progresso social. Desde então, a sua atuação se ampliou e, atualmente, também atua nas áreas de saúde, assistência, cultura, educação e lazer. O Sesc é organizado de forma federativa com um Departamento Nacional (DN) e os Departamentos Regionais (DDRR) nos estados. O DN normatiza, orienta e apoia as ações nos estados. Sua clientela preferencial é composta pelos comerciários e seus dependentes, principalmente os de menor renda, mas poderão ainda ser beneficiadas outras pessoas na categoria de usuário ou de participantes de projetos especiais. Dentro desta perspectiva, as escolas da RENEIF possuem a característica de ensino privado, com prioridade de atender aos filhos de comerciários com renda familiar de até três salários mínimos.

O presente estudo é do tipo transversal, de caráter descritivo e de censo composta pelos escolares da RENEIF. De acordo com dados da central de matrícula do Sesc, a população de escolares era, para 2012, de 32.283 alunos (15.735 na educação infantil e 16.548 na educação fundamental) matriculados em 117 escolas em 23 estados brasileiros e o Distrito Federal. Deste total, 34 escolas não participaram do presente estudo por dificuldades operacionais referentes a recursos humanos especializados com disponibilidade para treinamento para coleta de dados. Nas 83 escolas participantes, havia 28.853 escolares inscritos, o que representa $89,4 \%$ do total de crianças das 117 escolas do Sesc. Na região Sudeste só participaram duas escolas, ambas localizadas no estado do Espírito Santo.

Para participar, os pais/responsáveis dos alunos tinham que assinar e retornar um Termo de Consentimento Livre e Esclarecido (TCLE) que foi distribuído para todos os alunos das 83 escolas participantes. Todos os procedimentos da pesquisa e o TCLE foram aprovados pelo Comitê de Ética em Pesquisa do HUAP-UFF. Dos possíveis escolares a serem avaliados houve recusa de $30 \%$, totalizando 20.198 escolares participantes, o que representa $62,3 \%$ do total de inscritos nas escolas do Sesc em 2012. A grande maioria das 
A equipe responsável pela coleta dos dados foi composta por três nutricionistas do DN e 169 antropometristas, dentre estes, nutricionistas, pedagogos, enfermeiros e educadores físicos, todos do quadro do Sesc e treinados seguindo as mesmas técnicas de obtenção de medidas antropométricas. Os treinamentos foram realizados nos estados do Acre, Amazonas, Pará, Alagoas, Bahia, Ceará, Maranhão, Pernambuco, Distrito Federal, Goiás, Mato-Grosso, Espírito Santo, Paraná, Rio Grande do Sul e Santa Catarina. Nos demais estados participantes, os técnicos deslocaram-se para os estados próximos onde foram treinados com os técnicos locais previamente capacitados.

$\mathrm{O}$ treinamento das equipes foi realizado em dois momentos, no segundo semestre de $2011 \mathrm{e}$ no primeiro de 2012. Foram realizados seminários de padronização de medidas antropométricas, com conteúdos teóricos e práticos relativos à técnica de mensuração. A capacitação dos antropometristas foi avaliada pelo método de Habi$\mathrm{cht}^{14} \mathrm{e}$ os examinadores considerados aprovados em termos de precisão e exatidão na obteção dos dados participaram da coleta de dados.

\section{Coleta de Dados}

A coleta das medidas antropométricas de massa corporal e estatura foi realizada no período de março a junho de 2012 nas dependências das próprias escolas em dia e horário previamente agendados e seguiu as recomendações de Lohman et al. ${ }^{15}$. Foram agendadas também outras datas até duas semanas subsequentes, dependendo da possibilidade da equipe de coleta, para a realização das medições naquelas crianças que haviam faltado no dia originalmente agendado para a coleta de dados.

\section{Avaliação da massa corporal}

A medida de massa corporal (MC) foi obtida em balança digital portátil, da marca TANITA, modelo HS-302, com capacidade para $150 \mathrm{~kg}$ e precisão de $0,1 \mathrm{~kg}$. O procedimento foi realizado com os escolares vestindo roupas leves, descalços,

na posição ortostática (em pé e corpo ereto), com a MC distribuída em ambos os membros inferiores, braços soltos lateralmente ao corpo, ombros descontraídos e mantendo a cabeça erguida e olhando para frente. Foram feitas duas medições, sendo considerada a média dos valores para as análises.

\section{Avaliação da estatura}

Para a medida da estatura utilizou-se estadiômetro portátil, marca WISO, com campo de medição de $210 \mathrm{~cm}$, resolução em milímetros e que foi fixado em parede sem rodapé. O indivíduo foi posicionado com os braços ao longo do corpo, pés unidos e cabeça, nádegas e calcanhares encostados na parede, descalços e com a cabeça posicionada no plano de Frankfört. A régua do estadiômetro foi deslocada até a cabeça do avaliado e a leitura foi realizada em apneia após uma expiração normal. Foram feitas duas aferições da estatura sendo considerada a média dos valores para as análises.

\section{Determinação do estado nutricional}

Os dados foram digitados e armazenados em planilhas eletrônicas. O índice de massa corporal (IMC) foi calculado pela divisão da $\mathrm{MC}(\mathrm{kg})$ pelo quadrado da estatura $(\mathrm{m})$. $\mathrm{O}$ cálculo dos índices antropométricos Z estatura para idade (Est-I) e Z IMC para a idade (IMC-I) foi feito pelo programa Anthroplus ${ }^{16}$ disponível no site da OMS (http://www.who.int/growthref/tools/en/). Os dados de 85 crianças foram identificados como improváveis $(-6<$ Z Est-I $>+6$ ou $-5<$ Z IMC-I $>+5)^{13}$ o que demonstra a boa qualidade da medida antropométrica.

O diagnóstico nutricional foi feito baseandose nos valores de Z Est-I e Z IMC-I usando-se os padrões de referência sugeridos pela $\mathrm{OMS}^{16,17}$. Baixa estatura foi identificada nas crianças que apresentaram valores de Z Est-I $<-2$. Relativamente ao IMC-I, o estado nutricional foi identificado como: baixo IMC para idade (Z IMC-I < $-2)$; sobrepeso, para $+2<\mathrm{Z}$ IMC-I $\leq+3$ em crianças menores de 5 anos e, para as maiores, $+1<\mathrm{Z}$ $\mathrm{IMC}-\mathrm{I} \leq+2$; e obesidade, para Z IMC-I $>+3 \mathrm{em}$ crianças menores de 5 anos e, para as maiores, Z IMC-I > +2. Em determinados momentos, os valores de excesso de massa corporal (sobrepeso + obesidade) foram computados como o somatório na prevalência de sobrepeso e obesidade.

Estatística descritiva foi produzida com os dados antropométricos estratificados por carac- 
terísticas sociodemográficas (idade, sexo, macroregião de localização da escola).

\section{Resultados}

A maior quantidade de participação foi na faixa etária de 5 a 10 anos, que representa $56 \%$ do total de escolares. A faixa etária até 5 anos compreendeu $32 \%$ dos escolares e $12 \%$ tinham idade acima de 10 anos. A idade variou de 3 a 16,9 anos (Tabela 1). Os valores médios dos índices antropométricos Z Est-I e Z IMC-I foram positivos.

Para os escolares da RENEIF no Brasil como um todo, as medianas de estatura dos meninos praticamente se superpõem às das meninas até a faixa de 12 anos (Figura 1). As medianas tanto dos meninos quanto das meninas se assemelham às das mais recente POF 2008-2009.

Para o Brasil como um todo (Figura 1) e em todas as macroregiões, os escolares apresentaram crescimento linear adequado, semelhante ao padrão de referência proposto pela OMS. Por outro lado, as medianas do IMC dos escolares do RENEIF do Sesc se encontraram maiores do que os valores do padrão de referência, particularmente nos meninos (Figura 2B).

De fato, somente $1,6 \%$ das meninas e 1,3\% dos meninos tiveram baixa estatura (Tabela 2 ). Por outro lado, os resultados encontrados para as meninas foram de $6,9 \%$ de risco de sobrepeso, $17,5 \%$ de sobrepeso e $10,1 \%$ de obesidade (Tabela 2). Nos meninos, esses valores foram 7,$1 ; 17,6$ e $14,7 \%$, respectivamente. Deste modo, a prevalência de excesso de massa corporal encontrada foi de $30,0 \%$, para todas as crianças, sendo $27,6 \%$ em meninas e, um pouco mais elevado nos me$\operatorname{ninos}(32,3 \%)$.

Para as regiões, o percentual de sobrepeso variou de $23,5 \%$ para as meninas do Sudeste a
$15,5 \%$ para os meninos da região Sul (Tabela 2). Para obesidade, o maior percentual foi encontrado nos meninos do Nordeste e o menor nas meninas do Centro-Oeste. No caso de excesso de massa corporal, as maiores prevalências encontradas foram nas meninas do Sudeste e nos meninos do Nordeste e as menores prevalências foram encontradas nas meninas do Centro-Oeste e nos meninos do Sul.

Constatou-se que entre os escolares a prevalência de obesidade aumentou entre 3 a 4 vezes nos escolares com idade $>5$ anos comparativamente às mais novas (Tabela 3 ). Sem considerar a região Sudeste, pelo fato da baixa representação de escolares no grupo, a região Norte, para as meninas, apresentou os maiores aumentos proporcionais de obesidade dos mais novos para os mais velhos. Chama atenção a baixa prevalência de obesidade nas meninas $<5$ anos na região Norte. Nos meninos, é evidente a alta prevalência $(24,6 \%)$ de obesidade nos escolares com idade $\geq 10$ anos.

\section{Discussão}

Nos últimos anos houve mudança nos critérios de avaliação nutricional de crianças e adolescentes, particularmente no que diz respeito à referência a ser usada em todas as faixas etárias e os pontos de corte para os menores de 5 anos de idade $^{16,17}$. Assim, a comparação entre os resultados dos estudos deve sempre ser feita com cautela e deve ser concentrada em estudos que realizaram os mesmos procedimentos ${ }^{18,19}$.

$\mathrm{O}$ presente artigo constitui-se em relato dos resultados de estudo inédito nos escolares da RENEIF do Sesc que permitiu traçar a situação do crescimento e do estado nutricional antropométrico desta população. Os resultados indicam que o crescimento linear se encontra adequado,

Tabela 1. Média, desvio padrão (DP), valores mínimos e máximos da idade e medidas e índices antropométricos dos escolares, por sexo, da Rede Nacional de Ensino de Educação Infantil e Fundamental do Serviço Social do Comércio (Sesc), 2012.

\begin{tabular}{lrrrrrrrr}
\hline \multirow{2}{*}{ Variável } & \multicolumn{2}{c}{ Meninas } & \multicolumn{2}{c}{$(\mathbf{n}=\mathbf{9 . 9 9 2})$} & \multicolumn{2}{c}{ Meninos } & \multicolumn{2}{c}{$(\mathbf{n}=\mathbf{1 0 . 1 2 1})$} \\
\cline { 2 - 9 } & Média & \multicolumn{1}{c}{ DP } & Mínimo & Máximo & Média & \multicolumn{1}{c}{ DP } & Mínimo & Máximo \\
\hline Idade (anos) & 6,8 & 2,67 & 3,0 & 16,9 & 6,8 & 2,67 & 3,0 & 16,7 \\
Massa corporal $(\mathrm{kg})$ & 26,0 & 10,94 & 9,8 & 96,4 & 26,6 & 11,32 & 10,6 & 100,6 \\
Estatura $(\mathrm{cm})$ & 120,5 & 17,37 & 80,4 & 179,0 & 121,3 & 17,08 & 81,1 & 189,0 \\
IMC $\left(\mathrm{kg} / \mathrm{m}^{2}\right)$ & 17,2 & 2,88 & 9,9 & 37,3 & 17,4 & 2,90 & 10,8 & 35,3 \\
Z Est-I & 0,2 & 1,03 & $-4,3$ & 5,8 & 0,3 & 1,05 & $-3,6$ & 5,4 \\
Z IMC-I & 0,6 & 1,19 & $-4,9$ & 4,9 & 0,8 & 1,31 & $-4,4$ & 5,0 \\
\hline
\end{tabular}

IMC: Índice de Massa Corporal; IMC-I: Z IMC para Idade; Est-I : Z estatura para idade. 


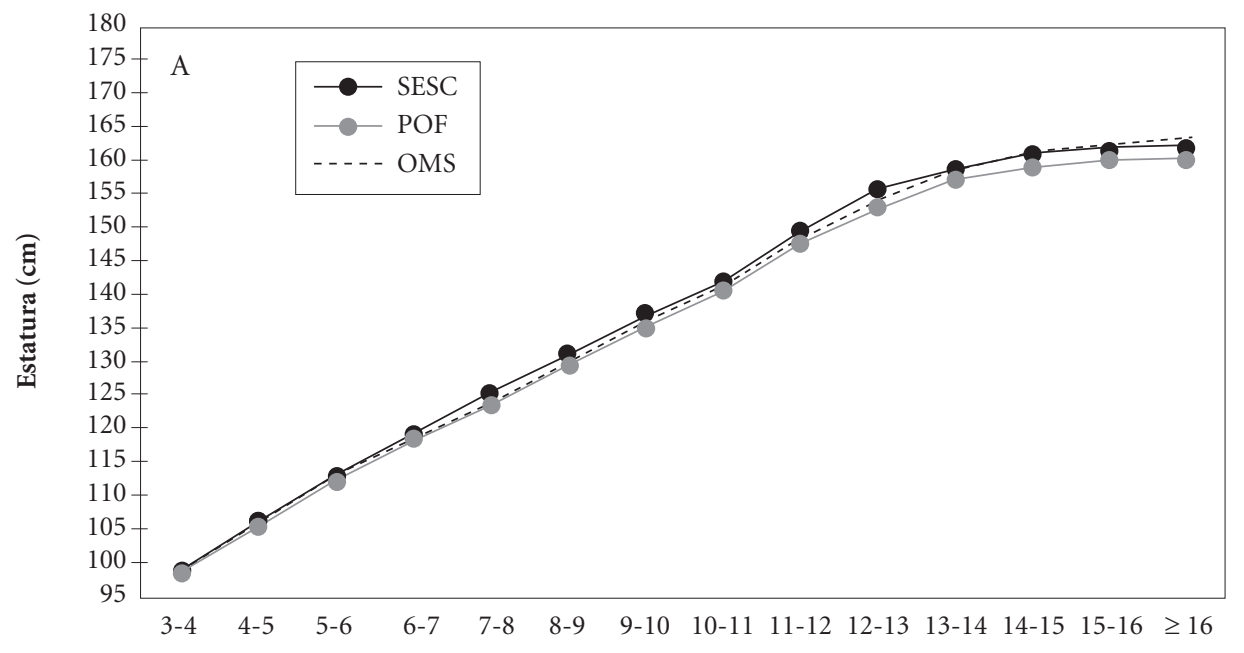

Faixa etária (anos)

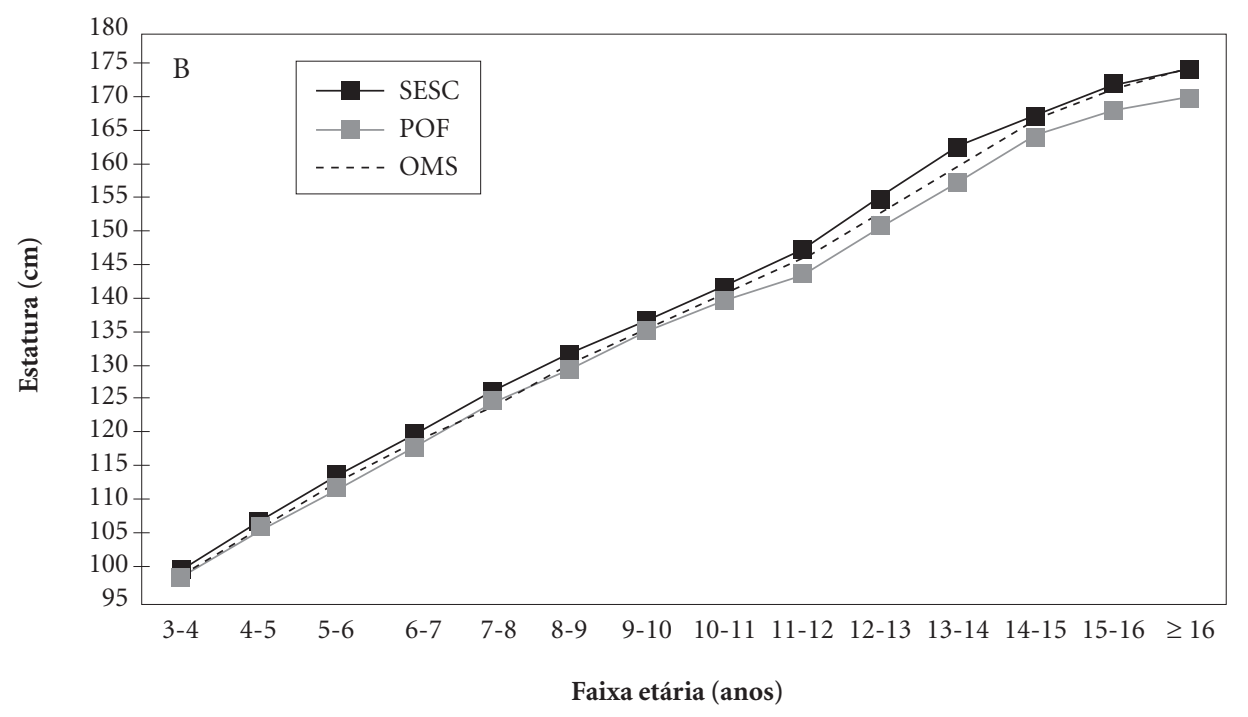

Figura 1. Medianas de estatura $(\mathrm{cm})$ em relação à faixa etária dos escolares da Rede Nacional de Ensino de Educação Infantil e Fundamental do Serviço Social do Comércio (Sesc), 2012 do sexo feminino (A) e masculino (B); da POF 2008-20091 e do padrão de referência da OMS ${ }^{13,16}$.

sendo semelhante ao padrão de referência proposto pela OMS em concordância com o quadro descrito para a população brasileira desta faixa etária como um todo ${ }^{1}$ e em escolares avaliados na Pesquisa Nacional de Saúde do Escolar (PenSe) $)^{11}$, realizada em amostra probabilística de escolares brasileiros de 13 a 15 anos em 2009. Assim, parece ser que a população de escolares brasileiros está expressando plenamente seu potencial de crescimento linear tanto em escolas públicas quanto particulares. Da mesma forma, em âmbito nacio- nal, a prevalência de baixo IMC-I foi baixa. Entretanto, os dados indicam uma prevalência alta de crianças com valores de IMC-I elevados.

Especificamente para as crianças até 5 anos de idade, observou-se alta proporção de risco de sobrepeso, sobrepeso e obesidade. Excesso de massa corporal nesta faixa etária foi semelhante ao encontado em âmbito nacional na POF de 2008-2009 (16,2\%) e sugere que essa situação já esteja ocorrendo precocemente na população brasileira $^{1}$. Baseando-se em dados provenientes 


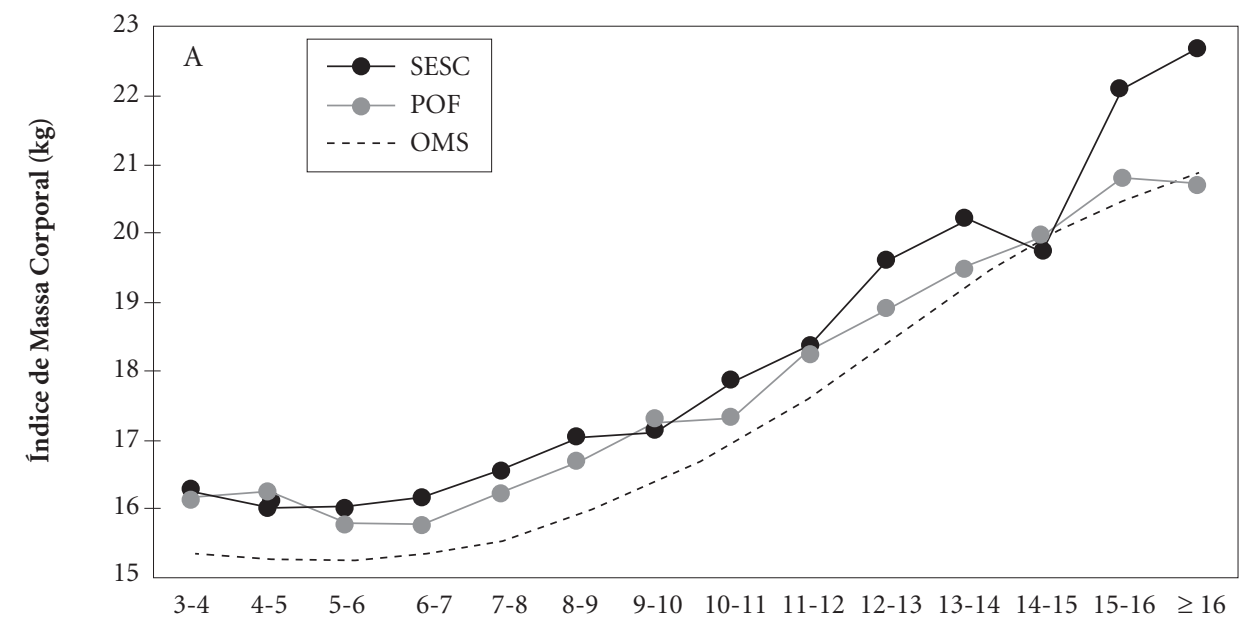

Faixa etária (anos)

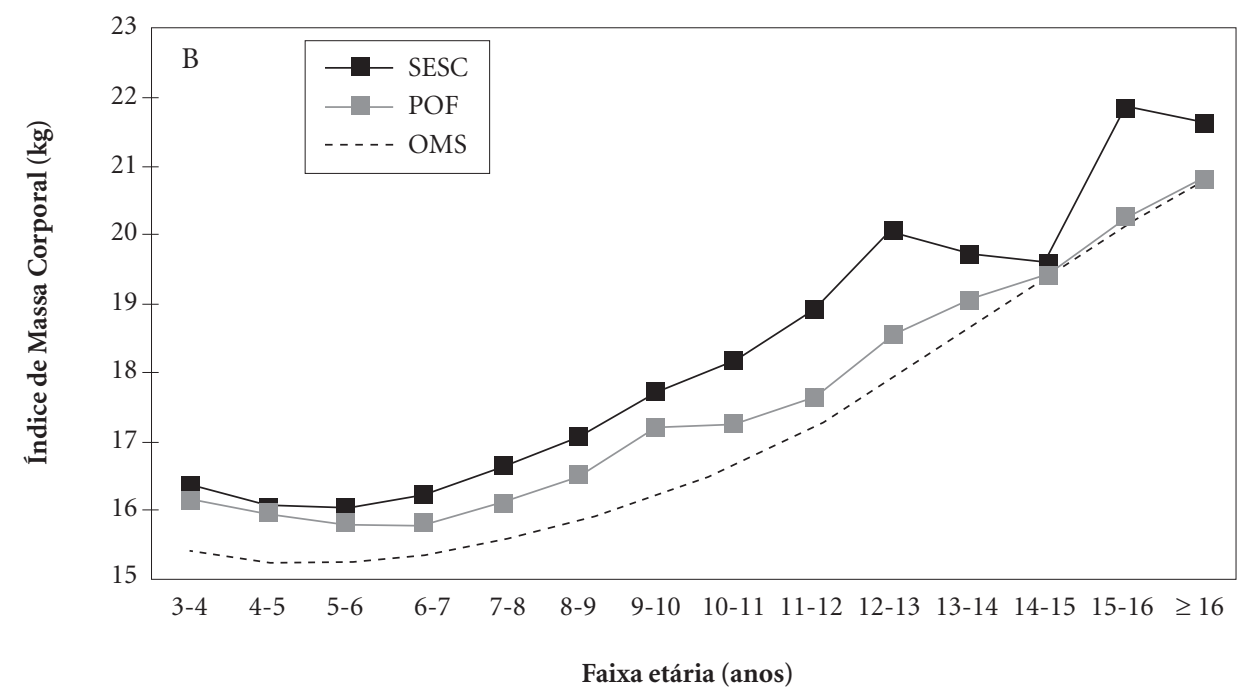

Figura 2. Medianas de índice de massa corporal $\left(\mathrm{kg} / \mathrm{m}^{2}\right)$ em relação à faixa etária dos escolares da Rede Nacional de Ensino de Educação Infantil e Fundamental do Serviço Social do Comércio (Sesc), 2012 do sexo feminino (A) e masculino (B); da POF 2008-20091 e do padrão de referência da OMS ${ }^{13,16}$.

de 450 estudos transversais em amostras representativas de 144 países em pré-escolares, 96,5\% dos quais tinham $<5$ anos de idade, De Onis et al. ${ }^{20}$ documentaram aumento na prevalência de excesso de massa corporal (Z IMC-I > 2) de 4,2\% em 1990 para 6,7\% em 2010. Globalmente, estima-se em $7 \%$ a prevalência de sobrepeso em crianças menores de 5 anos o que significa aumento de 54\% em relação às estimativas de 1990, com previsão de alcançar 9,9\% em $2025^{10}$. Entretanto, em outros lugares, já se evidencia redução da prevalência de obesidade nesta faixa etária. Dados recentes provenientes do National Health and Nutrition Examination Survey (NHANES), inquérito realizado periodicamente nos Estados Unidos, evidenciaram redução significativa de obesidade em crianças de 2 a 5 anos de 13,9\% para 8,4\% entre os ciclos de 2003-2004 para 2009-201021. Especificamente nos pré-escolares de baixa renda americanos, também observou-se redução pequena mas significativa na prevalência de obesidade em 19 dos 43 estados americanos investigados no Sistema de Vigilância Nutricional Pediátrica entre 2008 e $2011^{22}$.

Nas crianças mais velhas, de 5 a 10 anos de idade, a presente investigação também encontrou 
Tabela 2. Distribuição (\%) do estado nutricional pelos índices Z estatura para idade (Z Est-I) e Z índice de massa corporal para idade (Z IMC-I), segundo região e sexo, dos escolares da Rede Nacional de Ensino de Educação Infantil e Fundamental do Serviço Social do Comércio (Sesc), 2012.

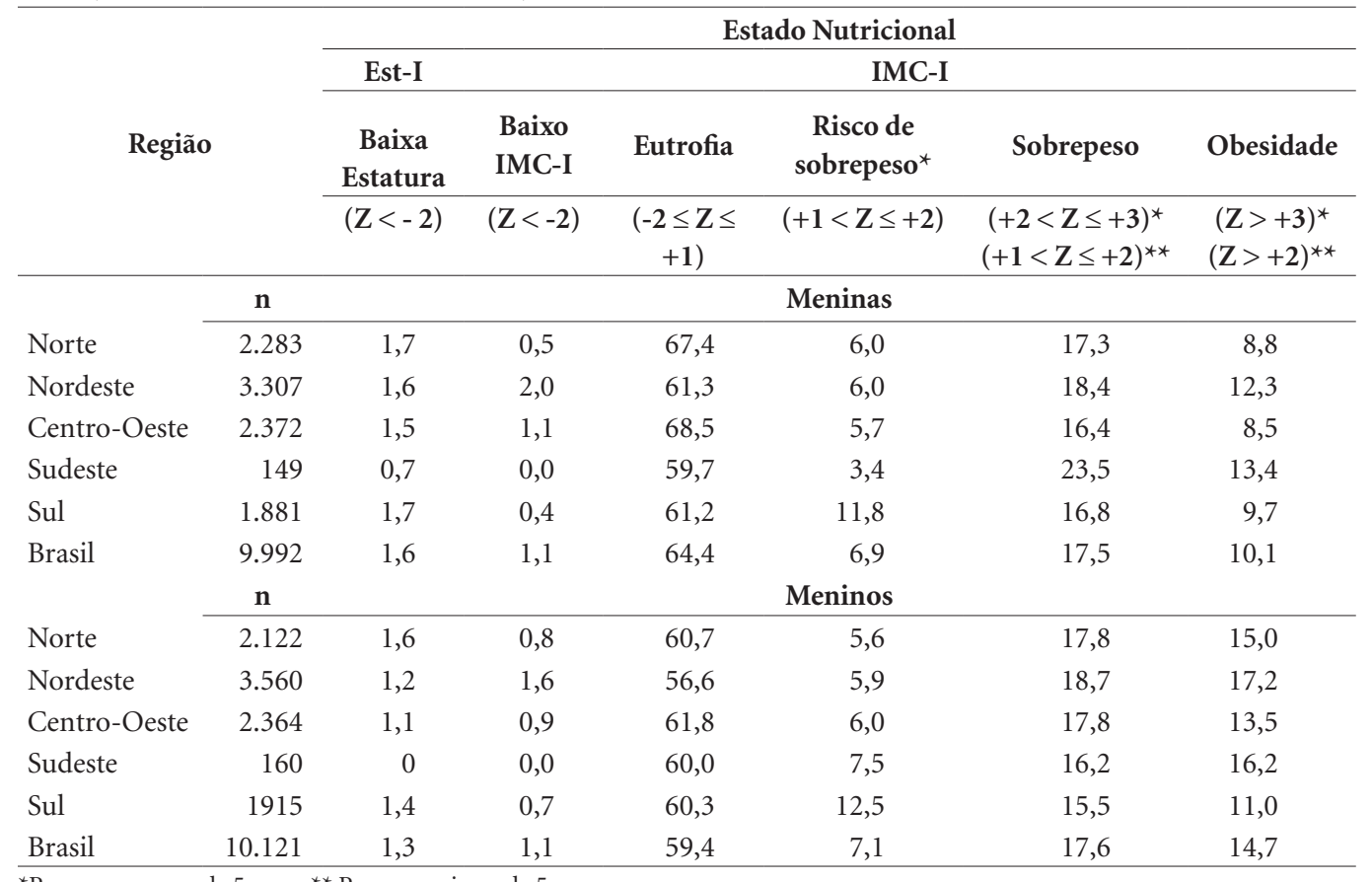

${ }^{\star}$ Para os menores de 5 anos; ${ }^{*}$ Para os maiores de 5 anos.

Tabela 3. Prevalência (\%) de risco de sobrepeso, sobrepeso e obesidade por faixa etária, segundo sexo e região, nos escolares da Rede Nacional de Ensino de Educação Infantil e Fundamental do Serviço Social do Comércio (Sesc), 2012.

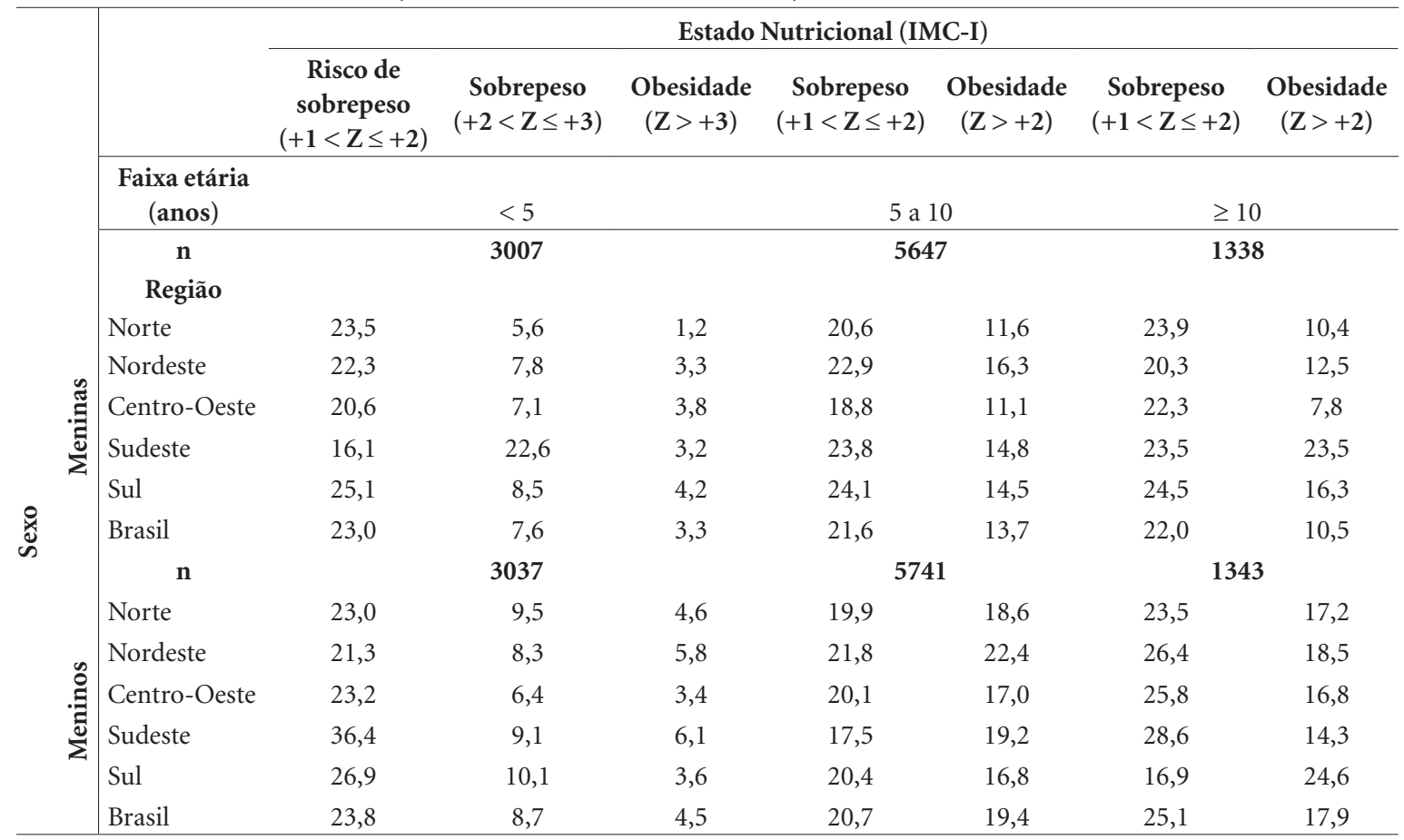

IMC-I: Índice de massa corporal para idade. 
prevalências tanto de excesso de massa corporal quanto de obesidade semelhantes às documentadas na POF 2008-2009 ${ }^{1}$. Relativamente às regiões do país, a prevalência de obesidade foi semelhante para meninos e meninas nas regiões Sul, Sudeste e Centro-Oeste comparativamente aos dados brasileiros observados na POF 2008-2009 mas para as regiões Norte e Nordeste os resultados encontrados neste estudo foram superiores.

Aparentemente, as prevalências de excesso de massa corporal nos escolares do Sesc na faixa etária de 5 a 10 anos se assemelham às encontradas em vários paíse europeus em estudos realizados pelo Departamento Europeu da Organização Mundial da Saúde, em $2006^{8}$, no qual as maiores prevalências foram encontradas em Portugal (31,5\% em crianças de 7 a 9 anos) e na Espanha (35,2\% em crianças de 2 a 9 anos). Em outros estudos do continente europeu, uma pesquisa com 1.225 escolares de 6 a 10 anos de idade, de ambos os sexos em Portugal em 2004, encontrou $35,6 \%{ }^{23}$ e na Noruega, no ano de 2007, em uma amostra composta por 955 escolares de 9 e 10 anos de idade, a prevalência foi de $20,3 \%{ }^{24}$.

Já para os adolescentes, como mostrado para as crianças até 10 anos de idade, evidencia-se, no Brasil como um todo, aumento continuado na prevalência de excesso de massa corporal ao longo dos quatro últimos inquéritos populacionais nacionais. No mais recente inquérito ${ }^{1}$, a prevalência foi de $21,5 \%$ para os meninos e $19,4 \%$ para as meninas, sendo de $20,5 \%$ no total da população entre 10 e 19 anos de idade. Em uma amostra grande de escolares brasileiros (36.976, sendo 20.914 meninos) de 10 a 15 anos conduzido entre 2004 e 2005 no Projeto Esporte Brasil ${ }^{25}$ encontrou-se prevalência de sobrepeso e obesidade de 11,9 e 2,6\%, respectivamente, com proporção mais elevada de sobrepeso nas meninas e de obesidade nos meninos, padrão semelhante ao observado no presente estudo.

Comparativamente aos resultados da PenSe ${ }^{11}$, que evidenciaram excesso de massa corporal em $23,2 \%$, sendo $7,2 \%$ a prevalência de obesidade, para ambos os sexos, os resultados encontrados nos escolares adolescentes do Sesc foram bem superiores. Tal achado pode estar relacionado à localização das escolas do SESC investigadas, situadas, em sua grande maioria, nos centros urbanos, capitais e cidades de médio porte, tendo assim, pouco representatividade da população rural. Deve-se atentar, também, ao fato de que uma grande parcela de alunos da rede serem oriundos da classe média, apesar dos valores cobrados serem acessíveis devido a subsídios. A ausência de análise por condições socioeconômicas, por questões operacionais, torna-se, portanto, uma limitação do presente estudo. Da mesma forma, para a região sudeste, apenas o estado do Espírito Santo com duas escolas com 311 crianças participaram no estudo, o que limita sua comparação com outras regiões. No caso específico de adolescentes, somente as escolas de Santa Catarina, com 3,9 \% da amostra, participaram na avaliação da região Sul.

O presente estudo tem pontos positivos mas algumas limitações. Primeiramente, o estudo foi realizado em um número total grande de escolares em 23 estados brasileiros e no Distrito Federal com um relativo baixo percentual de perdas, o que é difícil em inquéritos realizados neste segmento da população brasileira. Além disso, foram usadas técnicas padronizadas de medição antropométrica realizadas por profissionais da área da saúde ou pedagogos treinados por uma equipe central o que garantiu a qualidade das medidas e permitiu o diagnóstico nutricional neste segmento. Por outro lado, o estudo tem limitações. Num estudo deste porte sem financiamento externo, concentrou-se na realização do diagnóstico nutricional antropométrico dos escolares da RENEIF do Sesc o que não permitiu coletar dados mais detalhados sobre as características sociodemográficas, de ingestão alimentar, de prática de atividade física e de comportamento sedentário, fatores necessários para a explicação dos determinantes do quadro nutricional da população.

Os dados do presente estudo apontam para alta prevalência de obesidade o que indica estado de balanço energético (BE) positivo, ou seja, ingestão energética (IE) maior do que o gasto energético (GE). Como nenhum dos dois componentes foi medido no estudo, as interpretações dos resultados frente a possível causas do quadro só podem ser especulativas. Apesar da inexistência de dados objetivos nacionais sobre o $\mathrm{BE}^{26}$, existem dados recentes sobre IE da população brasileira ${ }^{27}$. Para a faixa etária de 10 a 18 anos, a IE média encontrada na Pesquisa de Orçamentos Familiares de 2008-2009 se encontrou sempre abaixo do recomendado usando os valores da $\mathrm{OMS}^{28}$, o que não era esperado frente ao quadro nutricional da população ${ }^{1}$. Estudos de IE na população em geral $^{29}$, mas particularmente em adolescentes ${ }^{30}$, são sempre um desafio para pesquisadores não sendo incomum o achado de baixa IE em obesos. Da mesma forma, é comum não se achar relação entre a obesidade e a prática de atividade física ${ }^{31}$ ou a inatividade física em adolescentes ${ }^{32}$. Assim, os estudos estão caminhando para a avaliação de 
padrões da dieta e estilos de vida saudáveis ${ }^{33,34}$ e indicam um quadro complexo na relação ${ }^{26,32,35,36}$.

Em suma, os dados sobre o estado nutricional dos escolares da RENEIF do Sesc demonstraram haver alta prevalência de sobrepeso e obesidade. Nesta perspectiva, pode-se considerar que os resultados deste estudo compõem um diagnóstico inicial da situação dos escolares e, apesar de não terem gerado informações sobre a causalidade do problema, indicam a necessidade de estratégias de abordagem multifocal, com integração das agendas dos diferentes setores, de modo a construir intervenções que articulem as dimen- sões de incentivo, apoio e proteção, necessárias à promoção da alimentação e vida saudáveis. Os resultados obtidos evidenciam a necessidade de implantação de um sistema de vigilância nutricional na RENEIF, para monitorar as modificações do estado nutricional desta população. Tal procedimento permitirá o acompanhamento sistemático com a finalidade de analisar a evolução do problema e as intervenções para a criação de ambientes saudáveis (escolas e comunidades), estratégia considerada como uma ação estruturante, que possibilita oportunidades práticas para a execução de estratégias integrais.

\section{Colaboradores}

LA Anjos e WDB Silveira participaram igualmente de todas as etapas da pesquisa: da concepção do estudo, do treinamento dos pesquisadores, analisaram os dados, escreveram o artigo e aprovaram sua versão final.

\section{Agradecimentos}

Os autores agradecem ao Sesc pelo apoio logístico e disponibilização do corpo docente e discente para a realização da pesquisa. Luiz Antonio dos Anjos recebeu bolsa de produtividade em pesquisa do CNPq.

\section{Referências}

1. Instituto Brasileiro de Geogafia e Estatística (IBGE). Pesquisa de Orçamentos Familiares POF 2008-2009. Antropometria e análise do estado nutricional de crianças, adolescentes e adultos no Brasil. Rio de Janeiro: IBGE; 2010.

2. Batista FM, Rissin A. A transição nutricional no Brasil: tendências regionais e temporais. Cad Saude Publica 2003; 19(Supl. 1):181-191.

3. Popkin BM. Contemporary nutritional transition: determinants of diet and its impact on body composition. Proc Nutr Soc 2011; 70(1):82-91.

4. World Health Organization (WHO). Preventing and managing the global epidemic: Report of WHO consultation group on obesity. Geneva: WHO; 2000. (WHO Technical Report Series, 894)

5. Anjos LA. Diagnóstico de obesidade e determinação de requerimentos nutricionais: desafios para a área de Nutrição. Cien Saude Colet 2013; 18(2):294.

6. Speakman JR. Obesity: the integrated roles of environment and genetics. J Nutr 2004; 134(Supl. 8):2090S-105S

7. Poirier P. Exploring the spectrum of diseases influenced by excess adiposity. Transl Res 2014; 164(4):278283.

8. World Health Organization (WHO). The challenge of obesity in the WHO European Region and the strategies for response. Summary. Copenhague: WHO Regional Office for Europe; 2007.

9. Anjos LA, Castro IRR, Engstrom EM, Azevedo AMF Crescimento e estado nutricional em amostra probabilística de escolares no Município do Rio de Janeiro, Brasil, 1999. Cad Saude Publica 2003; 19(Sup. 1):171179.

10. Black RE, Victora CG, Walker SP, Bhutta ZA, Christian P, de Onis M, Ezzati M, Grantham-McGregor S, Katz J, Martorell R, Uauy R. Maternal and child undernutrition and overweight in low-income and middle-income countries. Lancet 2013; 382(9890):427-451. 
11. Instituto Brasileiro de Geogafia e Estatística (IBGE). Pesquisa Nacional de Saúde do Escolar. Avaliação do estado nutricional dos escolares do $9^{\circ}$ ano do ensino undamental. Municípios das capitais e Distrito Federal. Rio de Janeiro: IBGE; 2009.

12. Serviço Social do Comércio. Carta da Paz Social, Documento Histórico do Sesc. Rio de Janeiro: Serviço Social do Comércio; 2012. [acessado 2015 mar 31]. Disponível em: http://www.sesc.com.br/wps/wcm/connect/490c55a9-a7c5-4a25-83a4-bb2f09491dea/Carta+da+Paz+Social.pdf?MOD=AJPERES\&CACHEID $=-$ 490c55a9-a7c5-4a25-83a4-bb2f09491dea

13. World Health Organization Multicentre Growth Reference Study Group (WHO). WHO Child Growth Standards: Length/height-for-age, weight-for-age, weight-forlength, weight-for-height and body mass index-for-age: Methods and development. Geneva: WHO; 2006.

14. Habicht JP. Estandartización de métodos epidemiológicos quantitativos sobre el terreno. Bol Oficina Sanit Panam 1974; 76(5):375-384.

15. Lohman TG, Roche AF, Martorell R. Anthropometric standardization reference manual. Champaign: Human Kinetics; 1988.

16. World Health Organization (WHO). WHO AnthroPlus for personal computers Manual: Software for assessing growth of the world's children and adolescents. Geneva: WHO; 2009.

17. de Onis M, Onyango AW, Borghi E, Siyam A, Nishida C, Siekmanna J. Development of a WHO growth reference for school-aged children and adolescents. Bull World Health Organ 2007; 85(9):660-667.

18. Gomes FS, Anjos LA, Vasconcellos MTL. Influence of different body mass index cut-off values in the assessment of nutritional status of adolescents in a household survey. Cad Saude Publica 2009; 25(8):1850-1857.

19. Gomes FS, Anjos LA, Vasconcellos MTL. Antropometria como ferramenta de avaliação do estado nutricional coletivo de adolescentes: uma revisão da literatura. Rev Nutr 2010; 23(4):591-605.

20. de Onis M, Blössner M, Borghi E. Global prevalence and trends of overweight and obesity among preschool children. Am J Clin Nutr 2010; 92(5):1257-1264.

21. Ogden CL, Carroll MD, Kit BK, Flegal KM. Prevalence of childhood and adult obesity in the United States, 2011-2012. JAMA 2014; 311(8):806-814.

22. Centers for Disease Control and Prevention (CDC).Vital Signs: Obesity Among Low-Income, Preschool-Aged Children - United States, 2008-2011. Morb Mortal Wkly Rep 2013; 62(31):629-634.

23. Ferreira RJ, Marques-Vidal PM. Prevalence and determinants of obesity in children in public schools of Sintra, Portugal. Obesity 2008; 16(2):497-500.

24. Oellingrath IM, Svendsen MV, Brantsæter AL. Eating patterns and overweight in 9- to 10-year-old children in Telemark County, Norway: a cross-sectional study. Eur J Clin Nutr 2010; 64(11):1272-1279.

25. Pelegrini A, Petroski EL, Coqueiro RS, Gaya ACA. Overweight and obesity in Brazilian schoolchildren aged 10 to 15 years: data from a Brazilian sports project. Arch Latinoam Nutr 2008; 58(4):343-349.
26. Mendonça CP, Anjos LA. Aspectos das práticas alimentares e da atividade física como determinantes do crescimento do sobrepeso/obesidade no Brasil. Cad Saude Publica 2004; 20(3):698-706.

27. Instituto Brasileiro de Geogafia e Estatística (IBGE). Pesquisa de Orçamentos Familiares 2008-2009. Análise do Consumo Alimentar Pessoal no Brasil. Rio de Janeiro: IBGE; 2011.

28. Food and Agriculture Organization of the United Nations (FAO), World Health Organization (WHO), United Nations (ONU). Expert Consultation: Human energy requirements. Rome: FAO; 2004. (Food and Nutrition Technical Report Ser, n.1).

29. Anjos LA, Souza DR, Rossato S. Desafios na medição da ingestão alimentar quantitativa de populações. Rev Nutr 2009; 22(1):151-161.

30. Santos LC, Pascoal MN, Fisberg M, Cintra IP, Martini LA. Notificação imprecisa da ingestão energética na dieta de adolescentes. J Pediatr 2010; 86(5):400-404.

31. Vasconcellos MB, Anjos LA, Vasconcellos MTL. Estado nutricional e tempo de tela de escolares do ensino fundamental do município de Niterói, RJ. Cad Saude Publica 2013; 29(4):713-722.

32. Coelho LG, Cândido APC, Machado-Coelho GLL, Freitas SN. Associação entre estado nutricional, hábitos alimentares e nível de atividade física em escolares. $J$ Pediatr 2012; 88(5):406-412.

33. Rodrigues PRM, Pereira RA, Cunha DB, Sichieri R, Ferreira MG, Vilela AAF, Gonçalves-Silva RM. Factors associated with dietary patterns in adolescents: A school-based study in Cuiabá, Mato Grosso. Rev Bras Epidemiol 2012; 15(3):662-674.

34. Azeredo CM, Rezende LFM, Canella DS, Claro RM, Castro IRR, Luiz OC, Levy RB. Dietary intake of Brazilian adolescents. Public Health Nutr 2015; 18(7):12151224.

35. Gonçalves H, González DA, Araújo CP, Muniz L, Tavares P, Assunção MC, Menezes AM, Hallal PC. Adolescents' perception of causes of obesity: unhealthy lifestyles or heritage? J Adolesc Health 2012; 51(6 Supl.):S46-S52.

36. Costa SM, Horta PM, Santos LC. Food advertising and television exposure: influence on eating behavior and nutritional status of children and adolescents. Arch Latinoam Nutr 2012; 62(1):53-59.

Artigo apresentado em 01/04/2015

Aprovado em 24/11/2015

Versão final apresentada em 26/11/2015 